\title{
Optoelectronic properties of hot-wire silicon layers deposited at $100{ }^{\circ} \mathrm{C}$
}

\author{
M. Brinza *, C.H.M. van der Werf, J.K. Rath, R.E.I. Schropp \\ Utrecht University, Faculty of Science, SID - Physics of Devices, P.O. Box 80 000, 3508 TA, Utrecht, The Netherlands
}

Available online 14 March 2008

\begin{abstract}
Hot-wire chemical vapor deposition is employed for the deposition of amorphous and microcrystalline silicon layers at substrate temperature kept below $100{ }^{\circ} \mathrm{C}$ with the aid of active cooling of the substrate holder. The hydrogen dilution is varied in order to investigate films at the amorphous-to-microcrystalline transition. While the amorphous layers can be produced with a reasonably low defect density as deduced from subgap optical absorption spectra and a good photosensitivity, the microcrystalline layers are of a lesser quality, most probably due to a decrease of crystallinity during the film growth. In the amorphous growth regime, the Urbach energy values decrease with increasing hydrogen dilution, reaching a minimum of $67 \mathrm{meV}$ just before the microcrystalline threshold. By varying the total gas pressure, the growth rate of the films is changed. The lowest deposition rate of this study $(0.16 \mathrm{~nm} / \mathrm{s})$ produced the amorphous sample with the highest photoresponse $\left(1 \times 10^{6}\right)$.
\end{abstract}

(C) 2007 Elsevier B.V. All rights reserved.

PACS: 78.66.Jg; 78.30.Ly; 73.61.Jc

Keywords: Silicon; Raman scattering; Conductivity; Microcrystallinity; Absorption; FTIR measurements; Photoconductivity; Raman spectroscopy

\section{Introduction}

The fabrication of solar cells on cheap plastic substrates like PET (polyester) would lead to an important reduction in the substrate-related production costs with the added advantage of lightweight and flexible modules. However, the use of cheap plastic substrates requires a substantial reduction in the substrate temperature to around $100{ }^{\circ} \mathrm{C}$ (PET for example can withstand temperatures up to $110^{\circ} \mathrm{C}$ ) while preserving the good optoelectronic properties of the thin silicon films. The applicability of hot-wire chemical vapor deposition (HWCVD) to depositions at such low substrate temperatures encounters a first obstacle in the form of considerable heating of the substrate due to the hot filaments. For the typical filament-substrate distance of $4 \mathrm{~cm}$ that is used in our hot-wire assembly in the deposition system PASTA, the substrate temperature stabilizes to $\sim 270^{\circ} \mathrm{C}$ without external substrate heating

\footnotetext{
${ }^{*}$ Corresponding author. Tel.: +31 302532345 .

E-mail address: m.brinza@phys.uu.nl (M. Brinza).
}

[1]. One way to reduce the heating effect of the hot-wires, which was already explored by our group [2], is to increase the filament-substrate distance. Indeed, an increase to $8 \mathrm{~cm}$ kept the substrate temperature below $100^{\circ} \mathrm{C}$ and allowed the deposition of amorphous films at a deposition rate of $0.11 \mathrm{~nm} / \mathrm{s}$ with a photoresponse $>10^{5}$ and $\mathrm{p}-\mathrm{i}-\mathrm{n}$ solar cells on Asahi $\mathrm{SnO}_{2}: \mathrm{F}$ coated glass (without $\mathrm{ZnO}$ at the back reflector) with an efficiency of $3.4 \%$. The present work deals with HWCVD silicon thin films deposited using an alternative method to maintain the desired substrate temperature, namely active cooling of the sample holder. The structural, optical and electrical properties of the silicon layers around the amorphous-to-microcrystalline transition are presented. It has long been known that a-Si:H films deposited just before the onset of crystallinity show the best properties in terms of stability against light induced degradation, low defect densities and improved medium-range order [3]. In the fabrication of layers at temperatures around $100{ }^{\circ} \mathrm{C}$, the use of hydrogen dilution becomes essential for reaching a reasonable quality for photovoltaic applications. 


\section{Experimental details}

A series of intrinsic layers were deposited in our multichamber ultra high vacuum system called PASTA, using a modified substrate holder which is actively cooled with flowing water at $14{ }^{\circ} \mathrm{C}$. Schott glass of $50 \mu \mathrm{m}$ thickness and c-Si wafers were used as substrates for single layers. For these substrates, temperature calibration performed in a 0.2 microbar Ar atmosphere showed that the equilibrium temperature on the side of the substrate facing the filaments is around $80^{\circ} \mathrm{C}$ and thus the substrate temperature during actual deposition could be kept below $100^{\circ} \mathrm{C}$. Moreover, the film growth starts at temperatures slightly lower than the stabilized temperature.

The hydrogen dilution $R$, defined as the ratio of the hydrogen and the silane flows, $R=\left[\mathrm{H}_{2}\right] /\left[\mathrm{SiH}_{4}\right]$, and the total process gas pressure $P_{\mathrm{g}}$ in the hot-wire reactor were the deposition parameters varied from sample to sample. For the first series of samples, the $P_{\mathrm{g}}$ was fixed at 50 microbar, the $\left[\mathrm{H}_{2}\right]$ flow was kept constant at $100 \mathrm{sccm}$ and the $\left[\mathrm{SiH}_{4}\right]$ was varied such that $R$ changed from 0 to 50 . For every hydrogen dilution, two different runs produced thin samples with thicknesses in the range $200-400 \mathrm{~nm}$ and thick samples with thicknesses between 700 and $1300 \mathrm{~nm}$. Two additional samples were deposited at $P_{\mathrm{g}}$ of 20 microbar with a thickness of $1000 \mathrm{~nm}$ and 75 microbar (2000 nm thickness) and $R=20$. A second dilution series was deposited at a $P_{\mathrm{g}}$ of 100 microbar and hydrogen dilution between 12 and 36 with sample thickness around $2000 \mathrm{~nm}$. For the latter series, the $\left[\mathrm{SiH}_{4}\right]$ flow was kept constant at $5 \mathrm{sccm}$ and the $\left[\mathrm{H}_{2}\right]$ flow was varied. Samples thicker than $700 \mathrm{~nm}$ were supplied with Ag coplanar electrodes for electrical measurements.

Raman spectroscopy was used to check the crystallinity of the layers, quantitatively described by the Raman crystalline ratio defined as $R_{\mathrm{c}}=\left(I_{510}+I_{520}\right) /\left(I_{480}+I_{510}+I_{520}\right)$, with $I_{480}, I_{510}, I_{520}$ the intensities of the transverse optic (TO) mode in a-Si, grain boundaries and/or small grains, and c-Si, respectively. Raman spectra were recorded using the $514.5 \mathrm{~nm}$ line of a Spectra-physics $\mathrm{Ar}^{+}$laser, a Spex triple-grating monochromator and a Roper Scientific CCD camera. The optical absorption coefficient $\alpha$ was obtained in the higher energy range from reflection/transmission measurements. Photothermal deflection spectroscopy (PDS) and constant photocurrent method (CPM) were used to measure the sub-band gap absorption. Photoconductivity was measured under AM1.5 illumination using a dual solar simulator (WACOM). FTIR spectra were recorded using a commercial FTIR spectrometer (Bio-Rad (Digilab) FTS40) on companion samples deposited on c-Si wafer.

\section{Results and discussion}

For the 50 microbar series, the first objective was to identify the dilution for which the amorphous to microcrystalline transition occurs at $100{ }^{\circ} \mathrm{C}$, since the best amorphous and microcrystalline materials are obtained close to the transition edge. Raman spectroscopy indicates the appearance of the $520 \mathrm{~cm}^{-1}$ peak associated with the crystalline Si TO mode for $R>25$. Concomitantly, the activation energy of the dark conductivity changes from $0.8 \mathrm{eV}$ to $0.5 \mathrm{eV}$ and the dark conductivity $\left(\sigma_{\mathrm{d}}\right)$ increases from $5 \times$ $10^{-11} \Omega^{-1} \mathrm{~cm}^{-1}$ to more than $10^{-7} \Omega^{-1} \mathrm{~cm}^{-1}$. The Tauc bandgap has values of $1.90-1.95 \mathrm{eV}$ for the amorphous layers while the refractive index at $630 \mathrm{~nm}, n_{630}$, equals $3.7 \pm 0.1$ for the best amorphous sample and decreases to 3.0 or lower for the microcrystalline samples. It is interesting to note that, in comparison with a previous series of samples deposited at $250^{\circ} \mathrm{C}$, where the transition was identified for $R \sim 20$ [4], higher dilution is required at $100^{\circ} \mathrm{C}$ to cross the amorphous to microcrystalline threshold.

The amorphous samples deposited before the transition show a photoresponse (defined as the ratio of photo- and dark conductivity) of $10^{5}$, as can be seen in Fig. 1. For the microcrystalline samples, the photoresponse drops to values lower than 10, which makes this material unsuitable for solar cells applications. One possible cause of the low photoresponse could be attributed to structural changes during film growth. Unlike PECVD $\mu \mathrm{c}-\mathrm{Si}: \mathrm{H}$, where the crystallinity of the layers increases with thickness, in HWCVD films a decrease of crystallinity with thickness is observed, not only at $100{ }^{\circ} \mathrm{C}$ but also at $250-270{ }^{\circ} \mathrm{C}$ substrate temperature $[1,4]$. Microcrystalline films grown close to the transition edge show the greatest difference in crystallinity. For example, for the $100{ }^{\circ} \mathrm{C}$ sample made at a hydrogen dilution of 30 , the Raman crystalline ratio amounts to $40 \%$ for a $200 \mathrm{~nm}$ thick sample and only $21 \%$ for a $1000 \mathrm{~nm}$ thick sample. This phenomenon, which can be related to filament ageing, was successfully counteracted for $\mu \mathrm{c}-\mathrm{Si}: \mathrm{H}$ solar cells grown at $270{ }^{\circ} \mathrm{C}$ by a reverse hydrogen profiling (increase

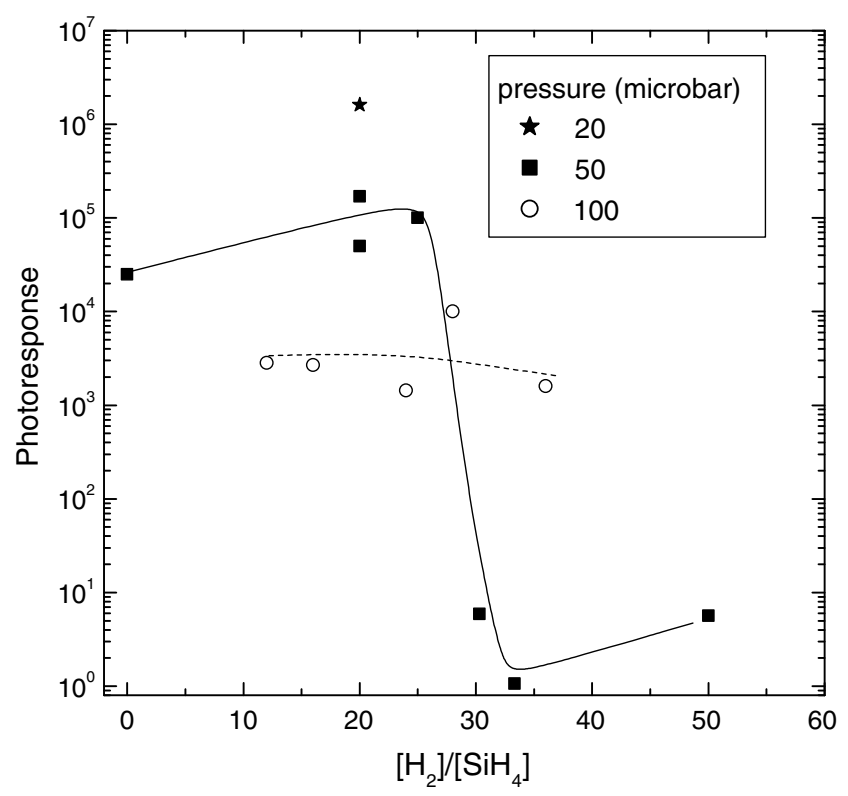

Fig. 1. Photoresponse versus hydrogen dilution for samples deposited at different pressures as indicated. The measurements were performed on samples with the thickness in the range $700-2000 \mathrm{~nm}$. The lines are guides to the eye. 


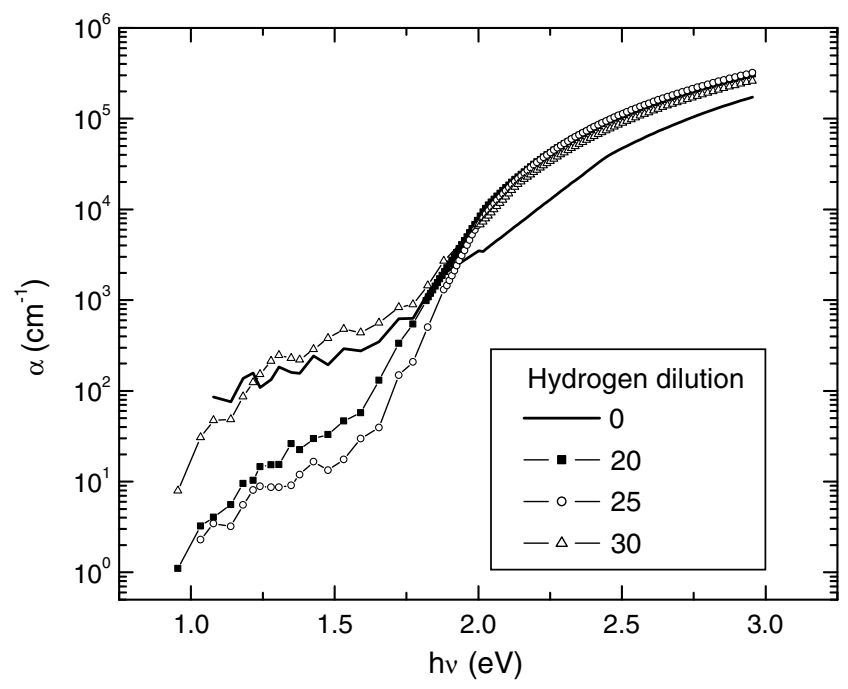

Fig. 2. Optical absorption of four samples deposited at 50 microbar pressure and hydrogen to silane ratios as given.

of the hydrogen dilution during deposition) [1]. Fig. 2 shows composite absorption coefficient spectra measured by CPM in the sub-bandgap energy range and scaled by $\alpha$ in the higher energy range, deduced from reflection/transmission measurements. Increasing the dilution in the amorphous growth regime has a positive effect on the quality of the material, resulting in a decrease of both the Urbach energy $\left(E_{\mathrm{u}}\right)$ down to a minimum of $67 \mathrm{meV}$ and of $\alpha(h v<1.4 \mathrm{eV})$. A low $E_{\mathrm{u}}$ indicates improved short range order of the a-Si network, while the value of $\alpha\left(1.2 \mathrm{eV}\right.$ ) (in $\left.\mathrm{cm}^{-1}\right)$ is usually translated into the density of dangling bonds $\left(\mathrm{in}^{-3}\right.$ ) through a factor of $2-5 \times 10^{16}[5]$. Interestingly enough, the sample deposited at $R=30$ has an amorphous-like absorption spectrum, even if it belongs to the microcrystalline growth regime. This can be explained through the fact that the crystalline fraction of this sample, averaged over the whole thickness of the sample, is low enough not to significantly contribute to optical absorption. On the other hand, the crystalline fraction at a certain depth is sufficiently high to allow a microcrystalline-specific conduction channel, as it was observed from dark conductivity measurements with coplanar electrodes. Furthermore, the amorphous fraction of this sample has a very disordered network (high Urbach energy), which explains the low photoresponse mentioned above. In Fig. 3 one can notice that there is a gradual decrease of the deposition rate with the hydrogen dilution. Furthermore, the deposition rates between thick and thin samples are comparable, suggesting that the transition from crystalline to amorphous phase is not related to the deposition rate.

In order to optimize the deposition condition of the amorphous material, the influence of the gas pressure in the reactor on the material properties was also investigated. The deposition rate increases with increasing pressure, as can be inferred from Fig. 3. On the other hand, the optoelectronic properties of the layers are negatively affected by the higher deposition rate. The layer deposited at

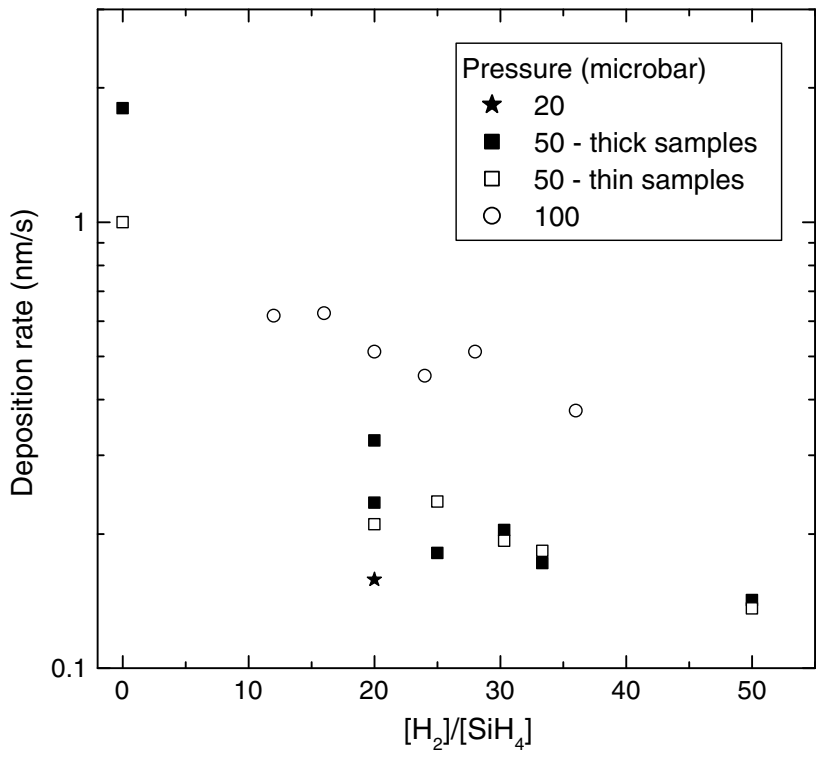

Fig. 3. Deposition rate versus hydrogen dilution for three different gas pressures.

20 microbar and $R=20$, with the lowest deposition rate of $0.16 \mathrm{~nm} / \mathrm{s}$, has the highest photoresponse of $1.6 \times 10^{6}$, an activation energy of the dark conductivity of $0.9 \mathrm{eV}$, $\sigma_{\mathrm{d}}$ of $5 \times 10^{-12} \Omega^{-1} \mathrm{~cm}^{-1}$ and an $E_{\mathrm{u}}$ of $68 \mathrm{meV}$. The Urbach energies of all amorphous-like films are summarized in Fig. 4. It is worth noticing that a similar $E_{\mathrm{u}}$ $(67 \mathrm{meV})$ was measured in a film grown at 50 microbar and higher hydrogen dilution $(R=25)$. However, there are differences in the absorption coefficients of the two samples below $1.4 \mathrm{eV}$ as measured by CPM and PDS. For the 20 microbar sample, PDS yields an $\alpha$ about 6 times higher than the $\alpha$ measured by CPM, while for the $P_{\mathrm{g}}=50$ microbar, $R=25$ sample, both CPM and PDS measure the same $\alpha$. The latter observation applies to all

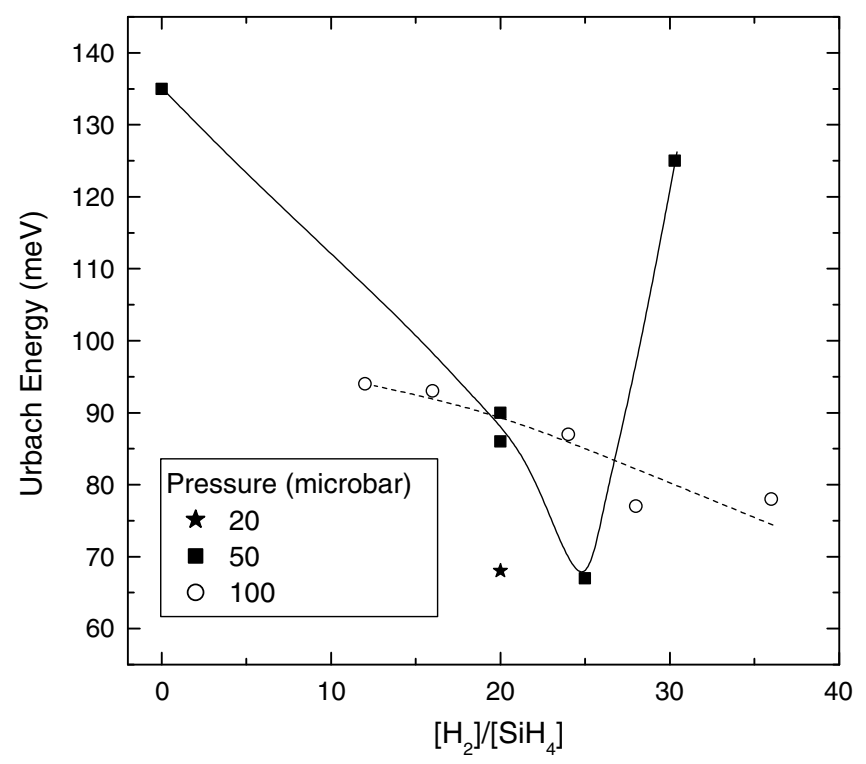

Fig. 4. Urbach energy versus hydrogen dilution for pressures as indicated. The lines are added as guides to the eye. 
samples deposited at a pressure of 50 microbar and higher. In low defect materials, it is expected that PDS measures slightly higher absorption coefficient beyond the Urbach tail, due to the fact that PDS detects all optical transitions, while CPM is not sensitive to transitions promoting free holes and to absorption at the surface and interface regions where there is enhanced recombination. Taking into account these considerations, one can conclude that in the $P_{\mathrm{g}}=50$ microbar, $R=25$ sample the bulk and the surface defect densities are of the same order of magnitude and that the transitions from occupied defects to the conduction band outnumber the transitions from the valence band to free defect states.

IR spectra give information about hydrogen bonding configuration in the silicon films. Fig. 5 shows such spectra for samples deposited at pressures of 20,50 and 75 microbar and $R=20$ hydrogen dilution. In the wavenumber interval $750-1200 \mathrm{~cm}^{-1}$, the doublet that appears at $845-860$ and $883-904 \mathrm{~cm}^{-1}$ is related to $\mathrm{SiH}_{2},\left(\mathrm{SiH}_{2}\right)_{n}$ and $\mathrm{SiH}_{3}$ vibrational modes [6]. The peaks at $1000-1100 \mathrm{~cm}^{-1}$ are usually assigned to $\mathrm{SiO}_{x}$ modes and indicate an open-structure material. One can note that this peak is barely detectable in the sample deposited at 50 microbar, which also had the highest refractive index $n_{630}=3.6$ of all three samples. By comparison, the 20 microbar sample has $\mathrm{n}_{630}=3.4$ and the 75 microbar sample has $n_{630}=3.1$. The other frequency range $1850-2300 \mathrm{~cm}^{-1}$ shown in Fig. 5 contains a first peak at $\sim 2000 \mathrm{~cm}^{-1}$ associated with the stretching mode of $\mathrm{SiH}$ and a second peak at $2090 \mathrm{~cm}^{-1}$ due to $\mathrm{SiH}_{2}$ stretching and clustered $\mathrm{SiH}$ at the inner surface of microvoids. The microstructure parameter $R^{*}$, defined as $R^{*}=I_{2090} /$ $\left(I_{2000}+I_{2090}\right)$, where $I_{x}=\int \alpha(\omega) / \omega \mathrm{d} \omega$, is another indicator of the layer quality; high $R^{*}$ values point towards void-rich materials. $R^{*}$ increases with the pressure from 0.29 in the 20 microbar sample to 0.32 in the 50 microbar sample and 0.48 in the 75 microbar sample. Moreover, in the 75 microbar sample, other peaks appearing at $\omega>2100 \mathrm{~cm}^{-1}$ are attributed to various $\mathrm{Si}-\mathrm{H}-\mathrm{O}$ bonding configurations. Samples deposited at 100 microbar have IR spectra similar to the 75 microbar sample. Hydrogen dilution within this series does not change substantially the IR spectra.

In the 100 microbar series, one can note that the amorphous growth regime is extended to even higher hydrogen dilution. Indeed, at $R=36$ the material is still amorphous while for the 50 microbar series the threshold is at $R>25$. Another cause of this delayed transition can lie in the fact that the dilution was changed by varying the hydrogen flow and keeping the silane flow constant for the 100 microbar series and oppositely for the 50 microbar series. A similar observation concerning the increase of the crystallinity threshold with the increase in the silane flow was made in an extensive study of HWCVD films deposited at $240{ }^{\circ} \mathrm{C}$ around the amorphous-to-microcrystalline transition [7]. The photoresponse is more or less constant within the 100 microbar series, with values smaller than $10^{4}$, while the $E_{\mathrm{u}}$ shows an improvement from values higher than $90 \mathrm{meV}$ to values less than $80 \mathrm{meV}$ with increasing dilution. However, the electronic quality of these layers is inferior to that of layers deposited at lower pressures.

\section{Conclusions}

Good photoresponse values of $10^{5}-10^{6}$ were obtained for amorphous silicon films deposited at around $100{ }^{\circ} \mathrm{C}$
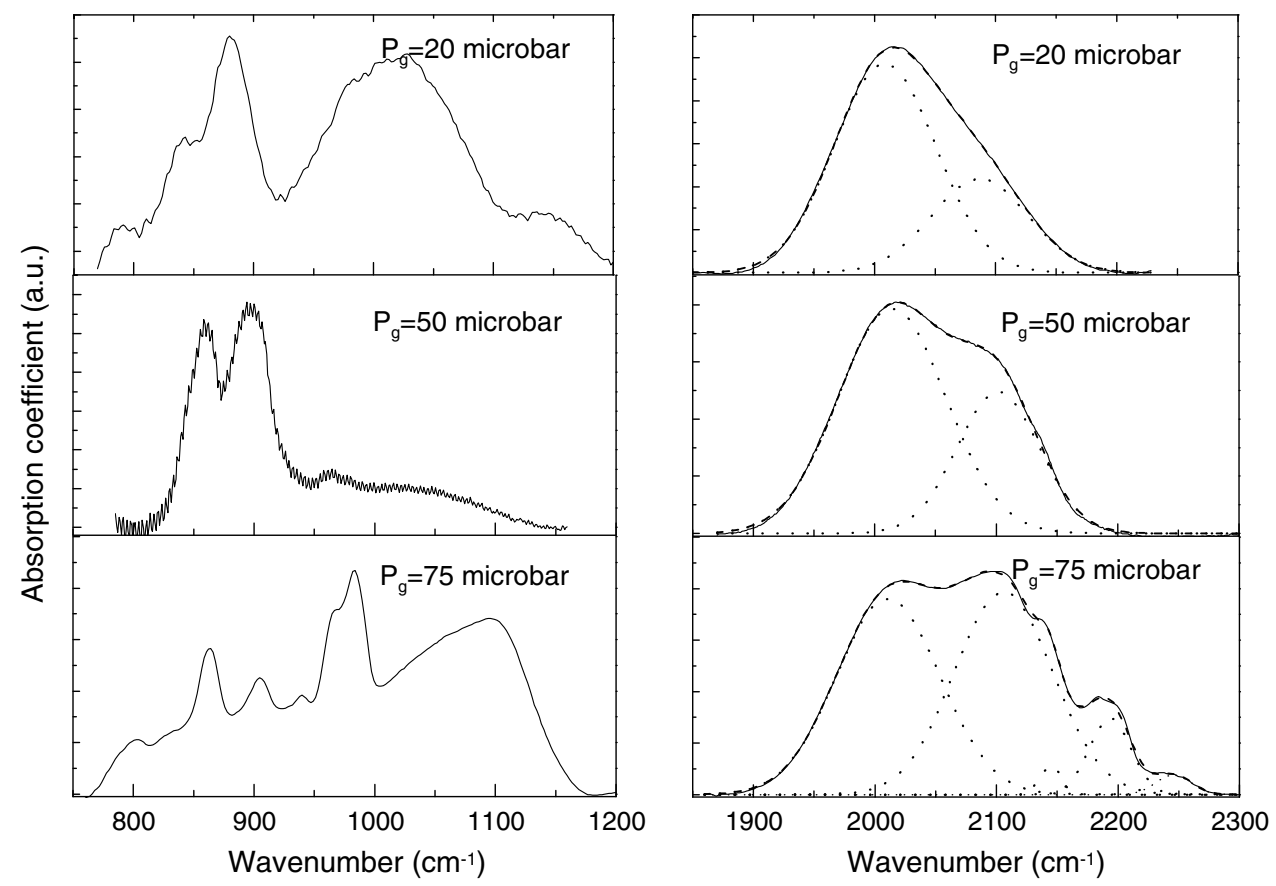

Fig. 5. FTIR spectra for two frequency ranges of $750-1200 \mathrm{~cm}^{-1}$ and $1850-2300 \mathrm{~cm}^{-1}$, measured in three samples deposited at a hydrogen dilution of 20 and gas pressure $P_{\mathrm{g}}$ as indicated. 
while an Urbach energy of $67 \mathrm{meV}$ and a dangling bond defect density of $6 \times 10^{16}-1 \times 10^{17} \mathrm{~cm}^{-3}$ deduced from CPM suggest moderate electronic quality. A lower deposition rate achievable by reducing the gas pressure proved to be beneficial for the overall performance of the layers, i.e. photoresponse, Urbach energy, deep-defect density and microstructure parameter. However, this improvement has to be reconciled with the presence of bonded oxygen, as detected by FTIR, which is usually related to the existence of microvoids in the film.

\section{Acknowledgement}

This project is undertaken with financial support from the Ministry of Economic Affairs of The Netherlands: program EOS (Subsidy Energy Research).

\section{References}

[1] H. Li, R.H. Franken, R.L. Stolk, C.H.M. van der Werf, J.K. Rath, R.E.I. Schropp, Thin Solid Films 516 (2008) 755.

[2] J.K. Rath, M. de Jong, R.E.I. Schropp, Thin Solid Films 516 (2008) 751.

[3] S. Guha, J. Yang, D.L. Williamson, Y. Lubianiker, J.D. Cohen, A.H. Mahan, Appl. Phys. Lett. 74 (1999) 1860.

[4] M. van Veen, PhD thesis, Utrecht University, 2003.

[5] N. Wyrsch, F. Finger, T.J. McMahon, M. Vanecek, J. Non-Cryst. Solids 137 (1991) 347.

[6] P.J. Zanzucchi, in: J.I. Pankove (Ed.), Hydrogenated amorphous silicon, Semiconductors and Semimetals, vol. 21, Academic, New York, 1984, p. 113, Part B.

[7] Daxing Han, J.D. Lorentzen, J. Weinberg-Wolf, L.E. McNeil, Qi Wang, J. Appl. Phys. 94 (2003) 2930. 